Original Article

\title{
Peiminine inhibits myocardial injury and fibrosis after myocardial infarction in rats by regulating mitogen-activated protein kinase pathway
}

\author{
Peng Chen", Dengming Zhou*, Yongsheng Liu, Ping Wang*, and Weina Wang* \\ Department of Vasculocardiology, Xiangyang Central Hospital, Affiliated Hospital of Hubei University of Arts and Science, Xiangyang, Hubei 441000, China
}

\section{ARTICLE INFO}

Received July 29,2021

Revised September 1, 2021

Accepted September 7, 2021

*Correspondence

Ping Wang

E-mail:wangping_0518@163.com

Weina Wang

E-mail: 115455396@qq.com

\section{Key Words}

Fibrosis

Mitogen-activated protein kinase

Myocardial infarction

Myocardial injury

Peiminine

\#These authors contributed equally to this work.

\begin{abstract}
Myocardial infarction promotes cardiac remodeling and myocardial fibrosis, thus leading to cardiac dysfunction or heart failure. Peiminine has been regarded as a traditional anti-fibrotic Chinese medicine in pulmonary fibrosis. However, the role of peiminine in myocardial infarction-induced myocardial injury and fibrosis remained elusive. Firstly, rat model of myocardial infarction was established using ligation of the left coronary artery, which were then intraperitoneally injected with 2 or $5 \mathrm{mg} / \mathrm{kg}$ peiminine once a day for 4 weeks. Echocardiography and haemodynamic evaluation results showed that peiminine treatment reduced left ventricular end-diastolic pressure, and enhanced maximum rate of increase/decrease of left ventricle pressure $( \pm \mathrm{dP} / \mathrm{dt} \max )$ and left ventricular systolic pressure, which ameliorate the cardiac function. Secondly, myocardial infarction-induced myocardial injury and infarct size were also attenuated by peiminine. Moreover, peiminine inhibited myocardial infarction-induced increase of interleukin (IL)- $1 \beta$, IL-6 and tumor necrosis factor- $\alpha$ production, as well as the myocardial cell apoptosis, in the rats. Thirdly, peiminine also decreased the myocardial fibrosis related protein expression including collagen I and collagen III. Lastly, peiminine reduced the expression of p38 and phosphorylation of extracellular signal-regulated kinase $1 / 2$ in rat model of myocardial infarction. In conclusion, peiminine has a cardioprotective effect against myocardial infarction-induced myocardial injury and fibrosis, which can be attributed to the inactivation of mitogen-activated protein kinase pathway.
\end{abstract}

\section{INTRODUCTION}

Myocardial infarction is a common and fatal cardiovascular disease all around the world [1]. Myocardial infarction enhances the apoptosis or necrosis of cardiomyocytes, which would progress to the loss of cardiomyocytes and further lead to congestive heart failure [2]. Ventricular remodeling and abnormal thickening of the cardiac muscles are the main progressive pathological process of myocardial infarction [3]. The ventricular remodeling leads to the excessive secretion of the extracellular matrixes, obstructs normal conduction of electrocardiogram, and reduces the ventricular compliance to induce myocardial fibrosis, thus resulting in myocardial fibrosis and sudden heart failure or cardiac death [4]. Therefore, myocardial fibrosis attenuation is considered an effective strategy to improve cardiac function post-myocardial infarction.

Mitogen-activated protein kinase (MAPK) cascades, including extracellular signal-regulated kinase 1/2 (ERK1/2), c-Jun NH(2)terminal kinase (JNK), and p38 MAPK, are highly conserved and play various roles in physiological processes [5]. MAPKs are found to be key genes that are associated with myocardial infarction [6]. MAPKs are involved in the cardiomyocyte apoptosis (i) (5) This is an Open Access article distributed under the terms of the Creative Commons Attribution Non-Commercial License, which permits unrestricted non-commercial use, distribution, and reproduction in any medium, provided the original work is properly cited. Copyright @ Korean J Physiol Pharmacol, pISSN 1226-4512, elSSN 2093-3827
Author contributions: P.C. and P.W. designed the study, supervised the data collection, D.Z. and Y.L. analyzed the data, interpreted the data, W.W. prepare the manuscript for publication and reviewed the draft of the manuscript. All authors have read and approved the manuscript. 
post myocardial infarction [7]. MAPKs also participate in the left ventricular remodeling process in rats suffering from myocardial infarction [8], activation of which can aggravate the myocardial infarction [9]. Suppression of MAPKs potentiates the protective effect on heart against myocardial infarction induced injury [10]. Therefore, regulating MAPKs pathway seems to be a promising strategy for treating myocardial infarction [11].

As a natural compound extracted from Fritillaria thunbergii Bulbi, peiminine has been widely used as a traditional Chinese medicine to treat various diseases, including cancer $[12,13]$ and atopic dermatitis [14]. Peiminine potentiates antioxidative [15], antiallergic [16] and anti-inflammatory [14] effects. Moreover, peiminine can attenuate inflammation-induced cell death in the dopaminergic neurons [17], facilitate lipopolysaccharide-induced mastitis by inhibiting ERK1/2 and p38 pathways [18]. In addition, peiminine has been reported to decrease the bleomycin-induced inflammatory response and pulmonary fibrosis in rats [19]. However, the role of peiminine in the cardiovascular diseases is still unclear. This study investigated the effects of peiminine on myocardial infarction-induced injury and fibrosis post-infarction, which might provide a novel strategy for the treatment of myocardial infarction.

\section{METHODS}

\section{Animal experiments}

Male Sprague-Dawley rats $(\mathrm{n}=24 ; 6-8$ weeks old and 220-250 g weight) were obtained from Jilin University Animal Center (Jilin, China) and housed in the cages under conditions $\left(23^{\circ} \mathrm{C} \pm\right.$ $3^{\circ} \mathrm{C}$ and $40 \%-60 \%$ humidity) with a 12 -h light/dark cycle. Ethi- cal approval was obtained from the Ethics Committee of Xiangyang Central Hospital, Affiliated Hospital of Hubei University of Arts and Science. Xiangyang Central Hospital, Affiliated Hospital of Hubei University of Arts and Science approved the protocol. This study was in accordance with the National Institutes of Health Laboratory Animal Care and Use Guidelines.

Rats were randomly separated into four groups: sham ( $\mathrm{n}=$ 6), myocardial infarction (MI, $\mathrm{n}=6$ ), MI treated with $2 \mathrm{mg} / \mathrm{kg}$ peiminine (Fig. 1A), and MI treated with $5 \mathrm{mg} / \mathrm{kg}$ peiminine. Rats in the MI groups were anaesthetized with $35 \mathrm{mg} / \mathrm{kg}$ sodium pentobarbital. A 14-gauge angiocath was endotracheally intubated into the rats with a ventilator. Hearts were exposed, and a 6-0 prolene suture was used to ligate the left coronary artery at 2-3 $\mathrm{mm}$ away from the origin between the left atrium and pulmonary artery conus. The occurrence of ST-segment elevation on electrocardiogram was used to identify the success of operation. Rats in the sham group were performed with thoracotomy and cardiac exposure, while without coronary ligation. Rats received an intramuscular injection of penicillin after the surgery. In peiminine treatment groups, 2 or $5 \mathrm{mg} / \mathrm{kg}$ peiminine (Gamma Technology Development Co., Ltd., Shenzhen, China) were intraperitoneally injected into the rats once a day for 4 weeks.

\section{Cardiac function assessment}

Rats were anesthetized with $10 \%$ chloral hydrate, and the right carotid artery was exposed after four weeks post peiminine treatment. A catheter tip micromanometer (Model SPR-838; Millar Instruments, Houston, TX, USA) was inserted into the left ventricular cavity. Maximum rate of increase/decrease of left ventricle pressure ( $\pm \mathrm{dP} / \mathrm{dt}$ max), left ventricular end-diastolic pressure (LVEDP) and left ventricular systolic pressure (LVSP)
A

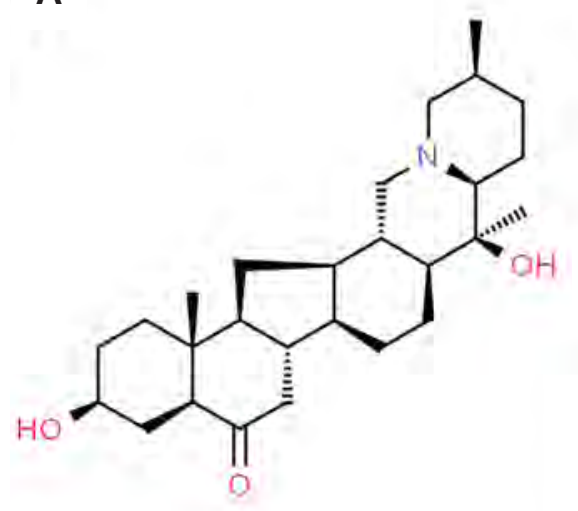

B

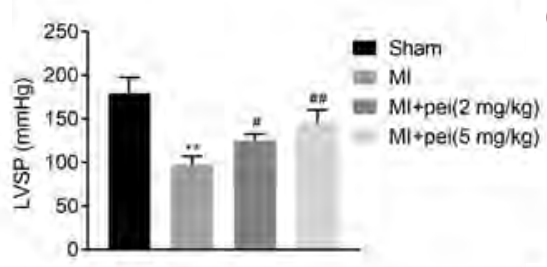

D

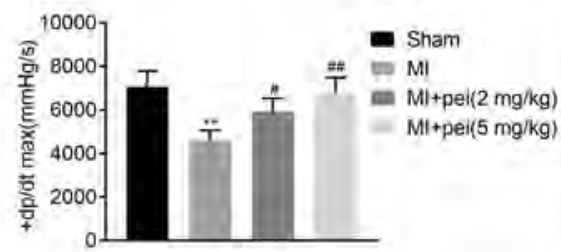

C

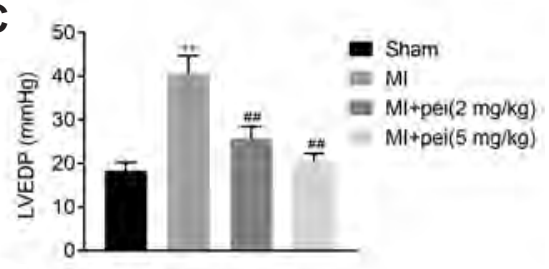

E

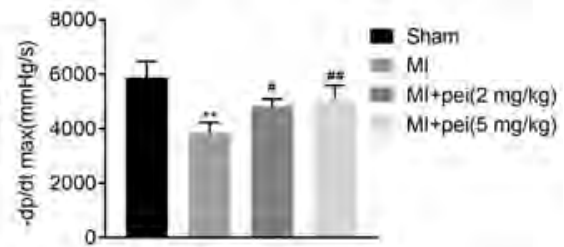

Fig. 1. Peiminine ameliorated cardiac function in myocardial infarction-induced rats. (A) Chemical structure of peiminine. (B) Peiminine administration attenuated myocardial infarction-induced decrease of left ventricular systolic pressure (LVSP) in rats. (C) Peiminine administration attenuated myocardial infarction-induced increase of left ventricular end-diastolic pressure (LVEDP) in rats. (D) Peiminine administration attenuated myocardial infarction-induced decrease of $+\mathrm{dP} / \mathrm{dt}$ max in rats. (E) Peiminine administration attenuated myocardial infarction-induced decrease of $-\mathrm{dP} / \mathrm{dt}$ max in rats. ${ }^{* *}$ vs. sham, $\mathrm{p}<0.01 .{ }^{\#, \# \#}$ vs. myocardial infarction (MI), $\mathrm{p}<0.05, \mathrm{p}<0.01$. 
were measured by the micromanometer.

\section{Histopathology}

Rats were euthanized and the heart tissues were harvested after four weeks post peiminine treatment. Following fixation in paraformaldehyde, the tissues were embedded in paraffin and cut into $4-\mu \mathrm{M}$ thickness sections. The sections were dewaxed and dehydrated, and then stained by hematoxylin and eosin (Sigma-Aldrich, Saint Louis, MO, USA) before observing using microscope (Olympus, Tokyo, Japan). The sections were also subjected to TTC and Masson's trichrome staining (Solarbio, Beijing, China). Images were captured under the microscope, and the infarct size and percentage of the fibrotic area were analyzed.

\section{TUNEL staining}

Following deparaffinization and rehydration, the heart sections were boiled in sodium citrate solution, and performed with TUNEL staining (Roche Diagnostics, Mannheim, Germany). The sections were stained with 4',6-diamidino-2-phenylindole, and then photographed with the microscope.

\section{ELISA}

Blood samples were collected from the abdominal aorta of rats, and then centrifuged at 3,000 $\mathrm{g}$ for $15 \mathrm{~min}$ to harvest the plasma. The level of lactate dehydrogenase (LDH), creatine kinase-MB (CK-MB), interleukin (IL)-1 $\beta$, IL-6 and tumor necrosis factor (TNF)- $\alpha$ in plasma was determined by commercial ELISA kits (RayBiotech, Inc., Norcross, GA, USA).

\section{Western blotting}

Myocardial tissues were lysed in radioimmunoprecipitation buffer (Beyotime, Shanghai, China). The protein supernatants were extracted from the homogenized tissues by centrifugation at $12,000 \mathrm{~g}$ for $1 \mathrm{~h}$. Protein concentration was determined using bicinchoninic acid method (Pierce, Rockford, IL, USA). Protein samples were performed with electrophoresis and transferred into polyvinylidene difluoride membranes (Millipore, Billerica, MA, USA). The membranes were blocked with 5\% fat-free milk and probed with primary antibodies against $\mathrm{Bax}(1: 2,000), \mathrm{Bcl}-2$ (1:2,000), collagen I $(1: 2,500)$, collagen III $(1: 2,500)$, p38 $(1: 3,000)$, p-p38 (1:3,000), ERK1/2 (1:3,500), p-ERK1/2 (1:3,500) and GAPDH $(1: 4,000)$ (Bioss, Beijing, China). Following incubation with horseradish peroxidase-conjugated immunoglobulin $\mathrm{G}$ (1:5,000; Abcam, Cambridge, MA, USA), the blots were detected by enhanced chemiluminescence (KeyGen, Nanjin, China).

\section{Statistical analysis}

Results from three independent experiments were presented as mean \pm SD. Statistical analysis between different groups were performed with one-way ANOVA or Student's t-test under SPSS 19.0 software (IBM Co., Armonk, NY, USA). p $<0.05$ was considered as significant difference.

\section{RESULTS}

\section{Peiminine ameliorated cardiac function in myocardial infarction rat model}

To assess the role of peiminine in myocardial infarction, rats post left coronary artery ligation were administered with peiminine. Cardiac function was investigated by haemodynamic evaluation. Results showed that LVSP (Fig. 1B) $\pm \mathrm{dP} / \mathrm{dt} \max$ (Fig. 1D, E) were decreased in myocardial infarction-rats, while LVEDP (Fig. 1C) was increased in rats post-myocardial infarction. These results indicated the myocardial infarction induced cardiac dysfunction in rat model. Peiminine administration enhanced LVSP (Fig. 1B) and $\pm \mathrm{dP} / \mathrm{dt} \max$ (Fig. 1D, E), while reduced LVEDP (Fig. 1C) and ameliorated the cardiac function in the myocardial infarction-induced rats, suggesting peiminine exhibited a cardioprotective effect against myocardial infarction induced injury.

\section{Peiminine ameliorated myocardial infarction-induced tissues damage in rats}

Hematoxylin-Eosin staining was performed to assess the histopathological changes. A normal cardiomyocyte structure was observed in the sham group (Fig. 2A). Left coronary artery ligation induced disturbances of cardiomyocyte, elicited inflammatory infiltration and disorganized structure in the heart tissues (Fig. 2A). However, peiminine administration alleviated the histopathological changes elicited by myocardial infarction (Fig. 2A). Moreover, TTC staining results exhibited more pale white areas in the heart of myocardial infarction-induced rats compared to sham group (Fig. 2B). Whereas, the viable areas were augmented by peiminine administration (Fig. 2B). Specific biomarkers for cardiac muscle damage including LDH and CK-MB were up-regulated in infarction-induced rats (Fig. 2C). Peiminine ameliorated myocardial infarction-induced tissues damage through downregulating plasma levels of $\mathrm{LDH}$ and $\mathrm{CK}-\mathrm{MB}$ (Fig. 2C) in rats.

\section{Peiminine ameliorated myocardial infarction-induced inflammation and apoptosis in rats}

Expression of proinflammatory factors such as IL-1 $\beta$, IL-6, and TNF- $\alpha$ were enhanced in infarction-induced rats, which were counteracted by peiminine administration (Fig. 3A), indicating 
A
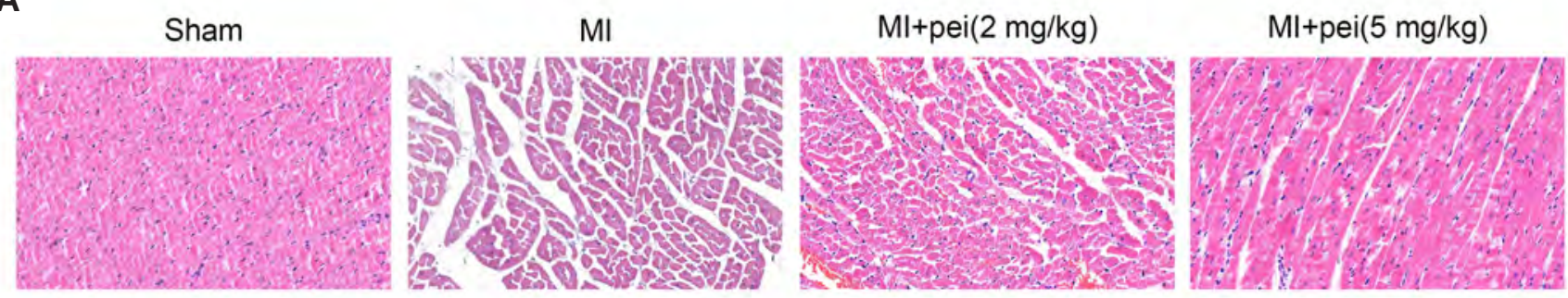

B
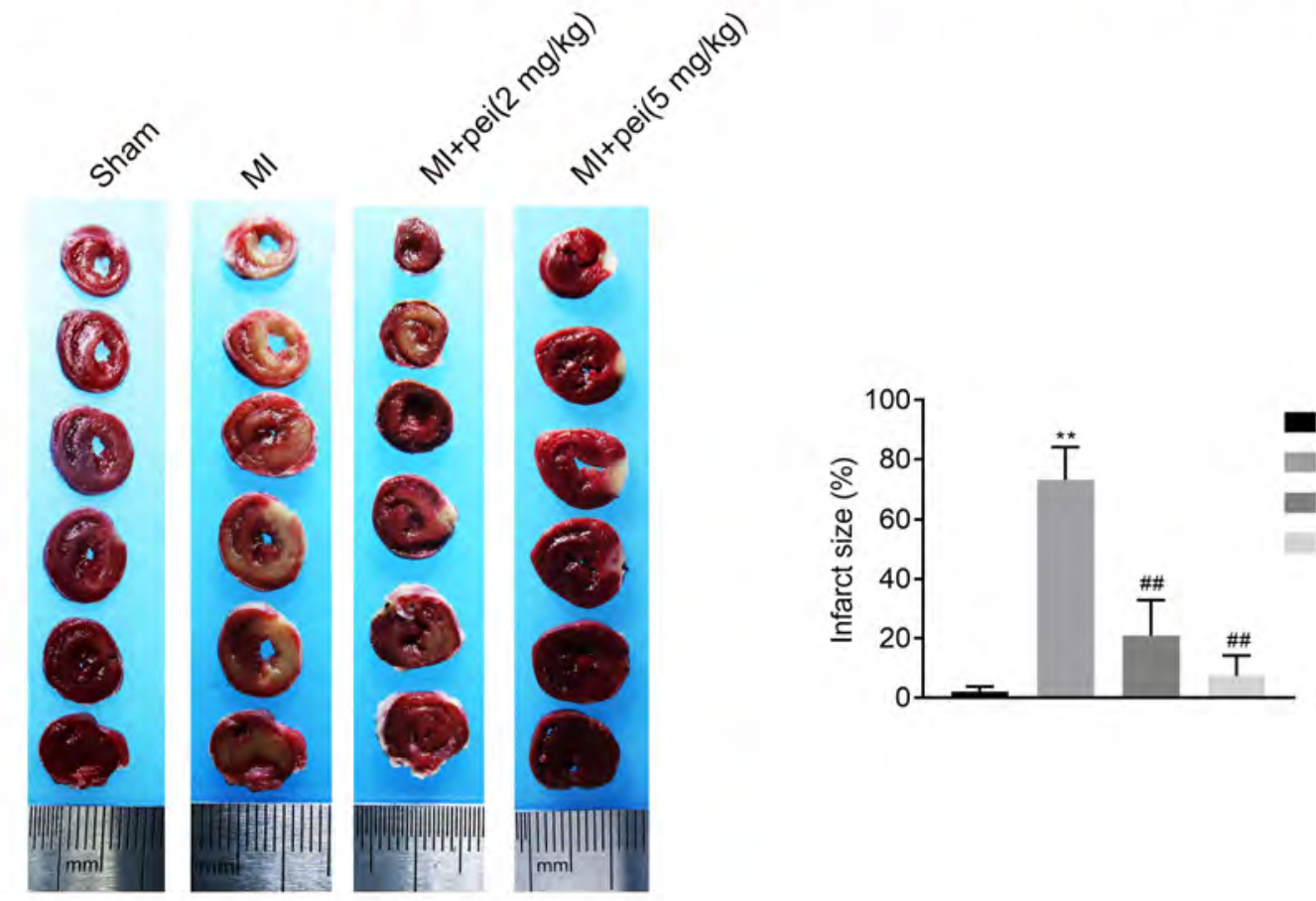

C
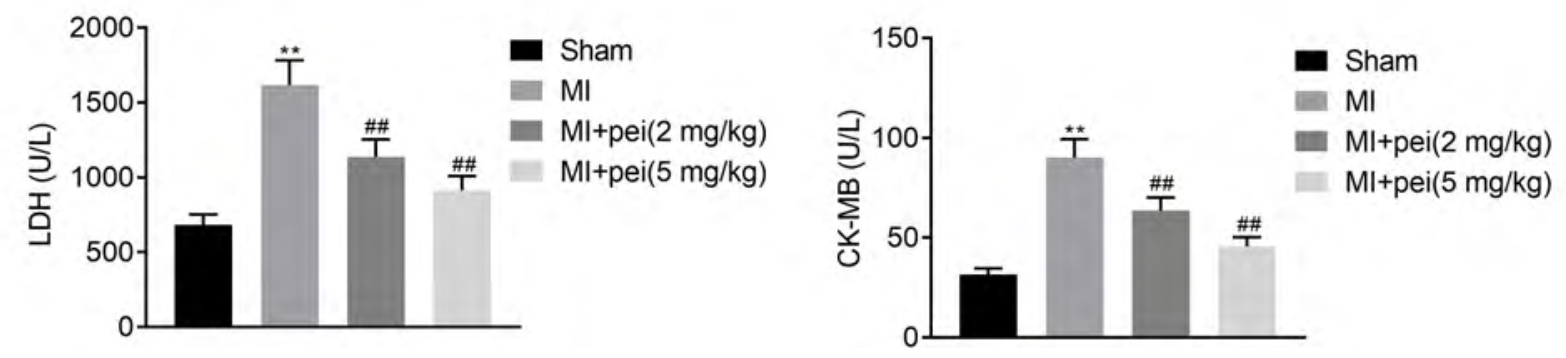

Fig. 2. Peiminine ameliorated myocardial infarction-induced tissues damage in rats. (A) Influence of left coronary artery ligation and peiminine administration on cardiomyocyte disturbances, inflammatory infiltration and structure of heart tissues detected by Hematoxylin-Eosin staining ( $\times 200)$. (B) Peiminine administration reduced myocardial infarction-induced increase of infarct size in rats detected by TTC staining. (C) Peiminine administration inhibited the myocardial infarction-induced increase of lactate dehydrogenase (LDH) in rats detected by ELISA. Peiminine administration inhibited the myocardial infarction-induced increase of creatine kinase-MB (CK-MB) in rats detected by ELISA. ${ }^{* *}$ vs. sham, $p<0.01 .{ }^{\# \#}$ vs. myocardial infarction (Ml), $\mathrm{p}<0.01$.

the anti-inflammatory effect of peiminine against myocardial infarction. Moreover, infarction-induced myocardial cell apoptosis (Fig. 3B), increase of Bax and decrease of Bcl-2 (Fig. 3C) were inhibited by peiminine administration, revealing the anti-apoptotic role of peiminine in myocardial infarction.

\section{Peiminine ameliorated myocardial infarction-induced fibrosis in rats}

Hearts exhibited more collagen fibers (blue) post myocardial infarction in MI group compared to the sham group (Fig. 4A). Peiminine decreased amount of the collagen fibers in rat model of myocardial infarction (Fig. 4A), suggesting the anti-fibrotic effect of peiminine against myocardial infarction. Moreover, peiminine 
A

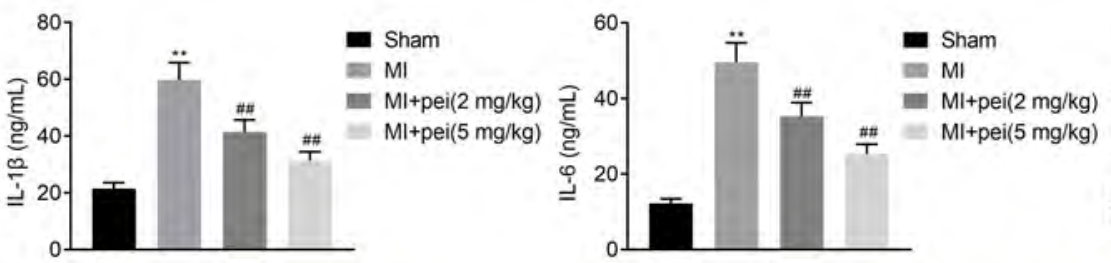

B

Sham

MI

$\mathrm{Ml}+\mathrm{pei}(2 \mathrm{mg} / \mathrm{kg})$
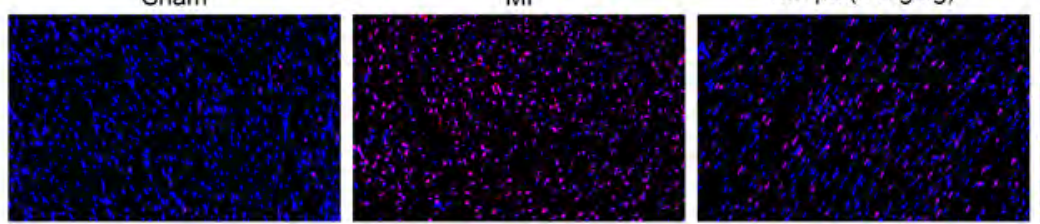

C
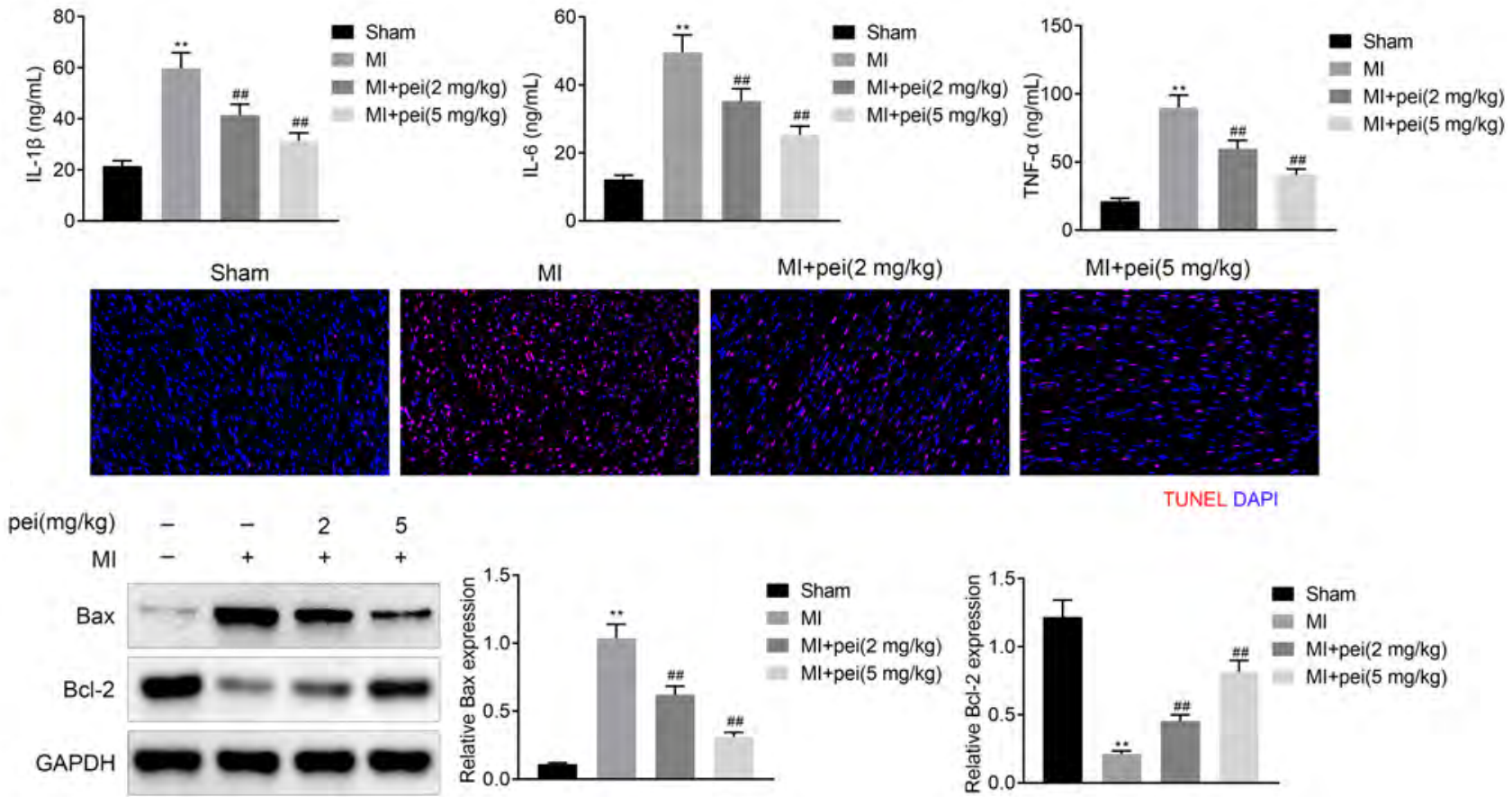

$\mathrm{Ml}+$ pei $(5 \mathrm{mg} / \mathrm{kg})$

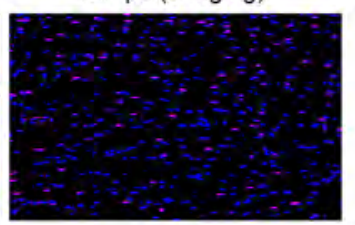

TUNEL DAPI

Fig. 3. Peiminine ameliorated myocardial infarction-induced inflammation and apoptosis in rats. (A) Peiminine administration suppressed the myocardial infarction-induced increase of CK-MB in rats detected by ELISA. (B) Peiminine administration inhibited myocardial infarction-induced increase of myocardial cell apoptosis in rats detected by TUNEL staining $(\times 200)$. (C) Peiminine administration inhibited the myocardial infarctioninduced increase of Bax and decrease of Bcl-2 in rats detected by Western blot. CK-MB, creatine kinase-MB; IL, interleukin; TNF, tumor necrosis factor. *** vs. sham, $\mathrm{p}<0.01 .{ }^{\# *}$ vs. myocardial infarction (MI), $\mathrm{p}<0.01$.

A

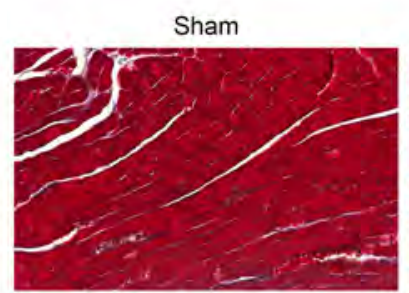

MI

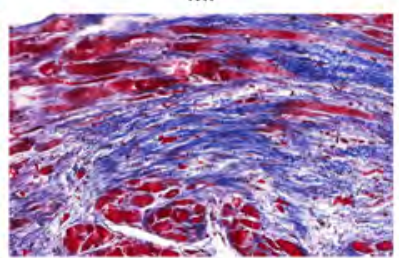

$\mathrm{Ml}+$ pei $(2 \mathrm{mg} / \mathrm{kg})$

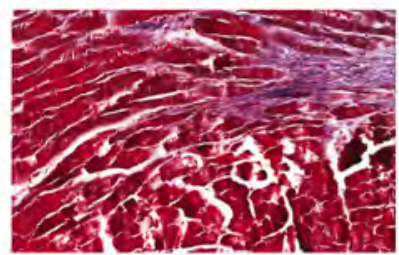

$\mathrm{Ml}+$ pei $(5 \mathrm{mg} / \mathrm{kg})$

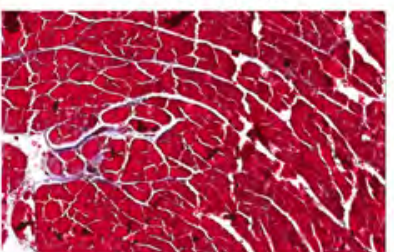

B

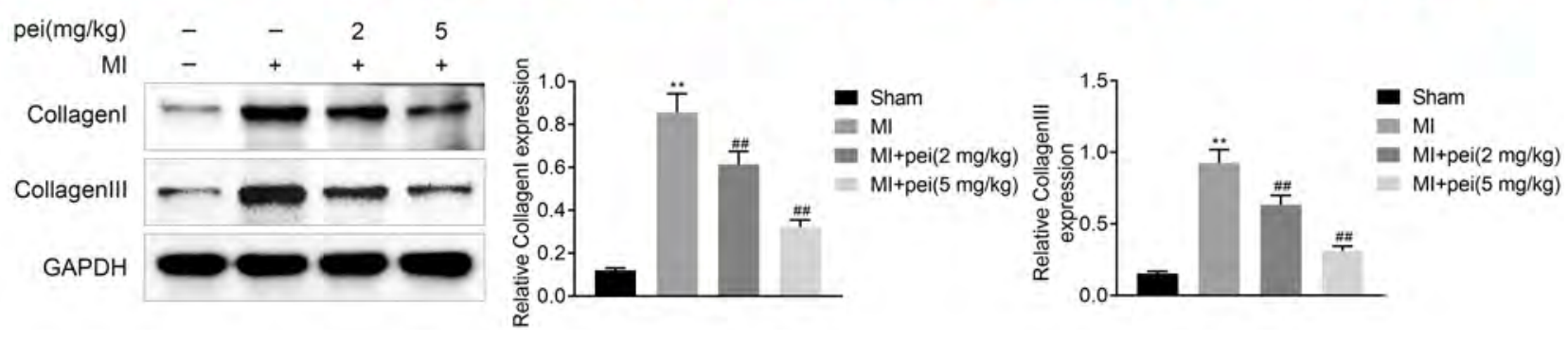

Fig. 4. Peiminine ameliorated myocardial infarction-induced fibrosis in rats. (A) Collagen fibers in hearts of rats post myocardial infarction in different groups. Masson's trichrome staining (×200). (B) Peiminine administration decreased the myocardial infarction-induced collagen I and III overexpression in rats detected by Western blot. ${ }^{* *}$ vs. sham, $\mathrm{p}<0.01$. ${ }^{\#}$ vs. myocardial infarction $(\mathrm{MI}), \mathrm{p}<0.01$.

counteracted the myocardial infarction-induced increase of collagen I and III protein expression (Fig. 4B), further confirming that peiminine ameliorated myocardial infarction-induced fibrosis in rats.

\section{Peiminine suppressed the activation of MAPKs in myocardial infarction-induced rats}

Protein expression of $\mathrm{p} 38$ and ERK1/2 were not affected by myocardial infarction (Fig. 5). In contrast, the p38 expression and 


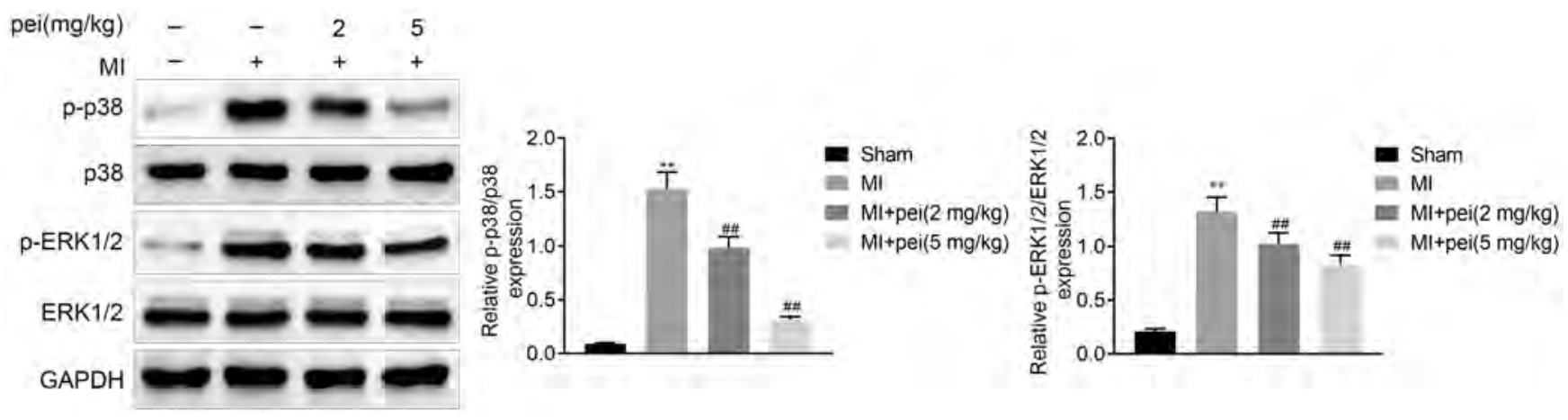

Fig. 5. Peiminine suppressed the activation of MAPKs in myocardial infarction-induced rats. p38 expression and ERK1/2 phosphorylation in different groups detected by Western blot. MAPK, mitogen-activated protein kinase; ERK1/2, extracellular signal-regulated kinase $1 / 2$. ${ }^{* *} v s$. sham, $\mathrm{p}<0.01$. \# vs. myocardial infarction (MI), $\mathrm{p}<0.01$.

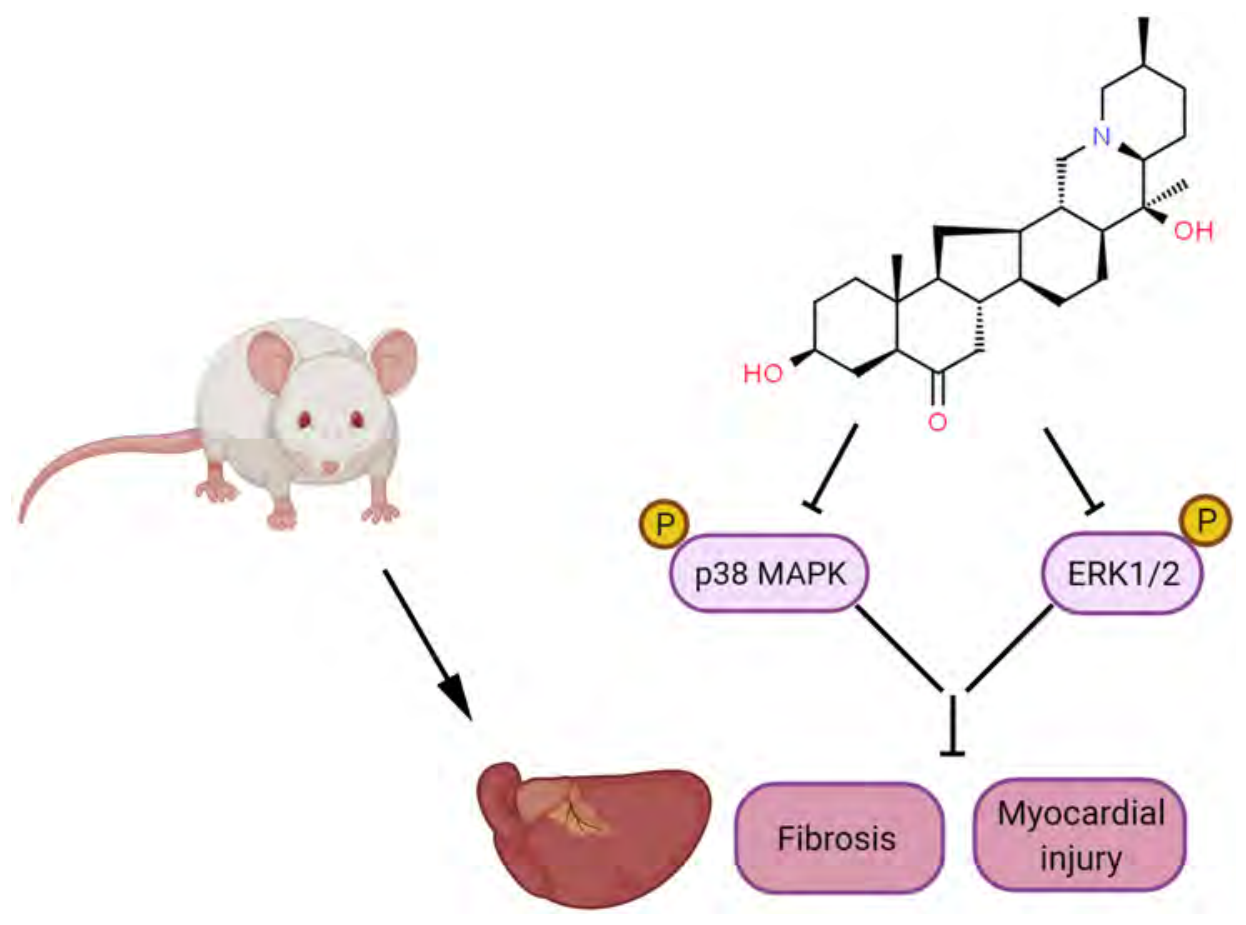

Fig. 6. Peiminine exhibited antiinflammatory, anti-apoptotic and anti-fibrotic activities to protect heart against from myocardial infarction through inactivation of MAPKs pathway. MAPK, mitogen-activated protein kinase; ERK1/2, extracellular signalregulated kinase $1 / 2$.
ERK1/2 phosphorylation were increased in rats post myocardial infarction, which was reduced by peiminine administration (Fig. 5), revealing that peiminine might exhibit cardioprotective effect through inactivating MAPKs pathway.

\section{DISCUSSION}

Myocardial infarction-induced injury in the cardiomyocytes is accompanied by a reparative fibrotic scar that prevents the rupture of ventricular wall [20]. However, excessive myocardial fibrosis might result in progressive cardiac dysfunction and lead to long-term heart failure [20]. Therefore, myocardial fibrosis is considered as the critical pathological change during the development of myocardial infarction-induced heart failure [21]. Several anti-fibrotic drugs, including angiotensin receptor blockers, angiotensin-converting enzyme inhibitors or calcium channel blockers, have been widely used in the clinical treatment of myocardial infarction [22]. However, the current anti-fibrotic drugs' long-term safety concerns and unsatisfactory efficacy necessitate identifying novel therapeutic strategies to protect against myocardial fibrosis [22]. Considering peiminine, the main active component of Fritillaria thunbergii Bulbi, has shown anti-fibrotic effect in pulmonary disease [19], the functional role and underlying molecular mechanism of peiminine in myocardial infarctioninduced myocardial fibrosis were investigated in this study.

Occlude of the left coronary artery is widely used for establishing the model of myocardial infarction [23]. Our results showed that left coronary artery ligation reduced LVSP and $\pm \mathrm{dP} / \mathrm{dt} \max$ but enhanced LVEDP to induce cardiac dysfunction. Moreover, TTC staining demonstrated that myocardial infarction-induced more pale white areas in the heart in MI group than that in the 
sham group. Hematoxylin-Eosin staining also confirmed the myocardial tissue damage caused by left coronary artery ligation. Peiminine administration ameliorated the cardiac dysfunction in myocardial infarction-induced rats, reduced the infarct size and attenuated the tissue damage, suggesting the cardioprotective role of peiminine against myocardial infarction.

Myocardial infarction-induced injury induces free radical generation, and triggers the release of TNF- $\alpha$ to initiate cytokine cascade and inflammatory response [24]. The immune cell infiltration caused by the inflammatory response is closely correlated with the myocardial cell apoptosis, thus impairing cardiac function during the development of myocardial infarction $[25,26]$. Suppression of apoptosis and inflammation post myocardial infarction attenuated the cardiac dysfunction [27]. Previous study has shown that inflammation-induced cell death of dopaminergic neurons was retarded by peiminine [17]. In this study, our results demonstrated that peiminine inhibited infarctioninduced increase of IL-1 $\beta$, IL- 6 , and TNF- $\alpha$, and suppressed the cell apoptosis with reduced Bax and enhanced Bcl-2 expression, suggesting the anti-inflammatory and anti-apoptotic effect of peiminine against myocardial infarction. However, inflammatory response exhibited protective role in the early stage of the myocardial infarction. Soon after coronary occlusion, the early anti-inflammatory strategy worsened the cardiac function and promoted myocyte apoptosis [28]. Therefore, the safety and the effectiveness of using peiminine to treat myocardial infarction should be further investigated.

Enhanced inflammatory response post myocardial infarction is found to be related to left ventricular remodeling, ventricular aneurysm formation and cardiac rupture, thus being concerned with fibrosis [29]. The fibrotic scar with excessive deposition of collagens has been shown to play an important role in the development of myocardial infarction [30]. Masson staining in this study indicated that myocardial infarction induced more collagen fibers accumulation in the hearts in MI group compared to the sham group. Peiminine administration reduced the collagen fibers and decreased collagen I and III protein expression, suggesting its anti-fibrotic role in myocardial infarction.

MAPKs pathway was implicated in the cardiac apoptosis and remodeling process of myocardial infarction [31]. Activation of MAPKs promotes the progression of acute myocardial infarction [32]. Peiminine has been reported to inhibit ERK1/2 and p38 pathway to relieve lipopolysaccharide-induced mastitis and bleomycin-induced acute lung injury. Here, increased p38 expression and ERK1/2 phosphorylation in rats post infarction was reversed by peiminine administration, revealing that peiminine might exhibit cardioprotective effect through inactivating MAPKs pathway. However, p38 MAPK was shown to confer pro-survival and cardioprotective effects on myocardial infarction through activation of $\alpha$-Crystallin B and Nrf2 [33]. The effect of peiminine on activating $\alpha$-Crystallin B and Nrf2 should be investigated to elucidate the controversial role of MAPKs on myocardial infarction.
In summary, as shown in Fig. 6, peiminine has been shown to exhibit anti-inflammatory, anti-apoptotic and anti-fibrotic activities to protect heart against from myocardial infarction. The cardioprotective mechanism of peiminine was partially mediated via inactivation of MAPKs pathway, revealing a new strategy of peiminine for treating the fibrotic diseases.

\section{FUNDING}

None to declare.

\section{ACKNOWLEDGEMENTS}

None.

\section{CONFLICTS OF INTEREST}

The authors declare no conflicts of interest.

\section{REFERENCES}

1. Jneid H, Alam M, Virani SS, Bozkurt B. Redefining myocardial infarction: what is new in the ESC/ACCF/AHA/WHF Third Universal Definition of myocardial infarction? Methodist Debakey Cardiovasc J. 2013;9:169-172.

2. Teringova E, Tousek P. Apoptosis in ischemic heart disease. J Transl Med. 2017;15:87.

3. Roever L, Palandri Chagas AC. Editorial: Cardiac remodeling: new insights in physiological and pathological adaptations. Front Physiol. 2017;8:751.

4. Gajarsa JJ, Kloner RA. Left ventricular remodeling in the postinfarction heart: a review of cellular, molecular mechanisms, and therapeutic modalities. Heart Fail Rev. 2011;16:13-21.

5. Çakır B, Kılıçkaya O. Mitogen-activated protein kinase cascades in Vitis vinifera. Front Plant Sci. 2015;6:556.

6. Qiu L, Liu X. Identification of key genes involved in myocardial infarction. Eur J Med Res. 2019;24:22.

7. Zhang Q, Lu L, Liang T, Liu M, Wang ZL, Zhang PY. MAPK pathway regulated the cardiomyocyte apoptosis in mice with postinfarction heart failure. Bratisl Lek Listy. 2017;118:339-346.

8. Matsumoto-Ida M, Takimoto Y, Aoyama T, Akao M, Takeda T, Kita T. Activation of TGF- $\beta 1$-TAK1-p38 MAPK pathway in spared cardiomyocytes is involved in left ventricular remodeling after myocardial infarction in rats. Am J Physiol Heart Circ Physiol. 2006;290:H709-H715.

9. Kumphune S, Bassi R, Jacquet S, Sicard P, Clark JE, Verma S, Avkiran M, O'Keefe SJ, Marber MS. A chemical genetic approach reveals that p38alpha MAPK activation by diphosphorylation aggravates myocardial infarction and is prevented by the direct binding of SB203580. J Biol Chem. 2010;285:2968-2975.

10. Zhang Z, Zhou S, Mei Z, Zhang M. Inhibition of p38MAPK poten- 
tiates mesenchymal stem cell therapy against myocardial infarction injury in rats. Mol Med Rep. 2017;16:3489-3493.

11. Bassi R, Heads R, Marber MS, Clark JE. Targeting p38-MAPK in the ischaemic heart: kill or cure? Curr Opin Pharmacol. 2008;8:141146.

12. Lyu Q, Tou F, Su H, Wu X, Chen X, Zheng Z. The natural product peiminine represses colorectal carcinoma tumor growth by inducing autophagic cell death. Biochem Biophys Res Commun. 2015;462:38-45.

13. Tang Q, Wang Y, Ma L, Ding M, Li T, Nie Y, Gu Z. Peiminine serves as an adriamycin chemosensitizer in gastric cancer by modulating the EGFR/FAK pathway. Oncol Rep. 2018;39:1299-1305.

14. Lim JM, Lee B, Min JH, Kim EY, Kim JH, Hong S, Kim JJ, Sohn Y, Jung HS. Effect of peiminine on DNCB-induced atopic dermatitis by inhibiting inflammatory cytokine expression in vivo and in vitro. Int Immunopharmacol. 2018;56:135-142.

15. Ruan X, Yang L, Cui WX, Zhang MX, Li ZH, Liu B, Wang Q. Optimization of supercritical fluid extraction of total alkaloids, peimisine, peimine and peiminine from the bulb of Fritillaria thunbergii Miq, and evaluation of antioxidant activities of the extracts. Materials (Basel). 2016;9:524.

16. Lee B, Kim EY, Kim JH, Min JH, Jeong DW, Jun JY, Cho CY, Sohn Y, Jung HS. Antiallergic effects of peiminine through the regulation of inflammatory mediators in HMC-1 cells. Immunopharmacol Immunotoxicol. 2015;37:351-358.

17. Chen G, Liu J, Jiang L, Ran X, He D, Li Y, Huang B, Wang W, Liu D, $\mathrm{Fu}$ S. Peiminine protects dopaminergic neurons from inflammation-induced cell death by inhibiting the ERK1/2 and NF- $\kappa \mathrm{B}$ signalling pathways. Int J Mol Sci. 2018;19:821.

18. Gong Q, Li Y, Ma H, Guo W, Kan X, Xu D, Liu J, Fu S. Peiminine protects against lipopolysaccharide-induced mastitis by inhibiting the $\mathrm{AKT} / \mathrm{NF}-\kappa \mathrm{B}, \mathrm{ERK} 1 / 2$ and $\mathrm{p} 38$ signaling pathways. Int J Mol Sci. 2018;19:2637.

19. Guo H, Ji F, Liu B, Chen X, He J, Gong J. Peiminine ameliorates bleomycin-induced acute lung injury in rats. Mol Med Rep. 2013;7:1103-1110.

20. Soriano FG, Guido MC, Barbeiro HV, Caldini EG, Lorigados CB, Nogueira AC. Endotoxemic myocardial dysfunction: subendocardial collagen deposition related to coronary driving pressure. Shock. 2014;42:472-479.

21. Liu JJ, Huang N, Lu Y, Zhao M, Yu XJ, Yang Y, Yang YH, Zang WJ. Improving vagal activity ameliorates cardiac fibrosis induced by angiotensin II: in vivo and in vitro. Sci Rep. 2015;5:17108.

22. Li C, Han R, Kang L, Wang J, Gao Y, Li Y, He J, Tian J. Pirfenidone controls the feedback loop of the AT1R/p38 MAPK/renin-angiotensin system axis by regulating liver $\mathrm{X}$ receptor- $\alpha$ in myocardial infarction-induced cardiac fibrosis. Sci Rep. 2017;7:40523.

23. Reichert K, Colantuono B, McCormack I, Rodrigues F, Pavlov V, Abid MR. Murine left anterior descending (LAD) coronary artery ligation: an improved and simplified model for myocardial infarction. J Vis Exp. 2017;(122):55353.

24. Frangogiannis NG, Smith CW, Entman ML. The inflammatory response in myocardial infarction. Cardiovasc Res. 2002;53:31-47.

25. Wan N, Liu X, Zhang XJ, Zhao Y, Hu G, Wan F, Zhang R, Zhu X, Xia H, Li H. Toll-interacting protein contributes to mortality following myocardial infarction through promoting inflammation and apoptosis. Br J Pharmacol. 2015;172:3383-3396.

26. Ge J, Guo K, Zhang C, Talukder M, Lv MW, Li JY, Li JL. Comparison of nanoparticle-selenium, selenium-enriched yeast and sodium selenite on the alleviation of cadmium-induced inflammation via

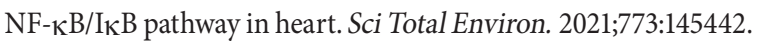

27. Fan D, Yang Z, Yuan Y, Wu QQ, Xu M, Jin YG, Tang QZ. Sesamin prevents apoptosis and inflammation after experimental myocardial infarction by JNK and NF-кB pathways. Food Funct. 2017;8:28752885.

28. Wang X, Guo Z, Ding Z, Mehta JL. Inflammation, autophagy, and apoptosis after myocardial infarction. J Am Heart Assoc. 2018;7:e008024.

29. Anzai T. Inflammatory mechanisms of cardiovascular remodeling. Circ J. 2018;82:629-635

30. Zhang X, Hu W, Feng F, Xu J, Wu F. Apelin-13 protects against myocardial infarction-induced myocardial fibrosis. Mol Med Rep. 2016;13:5262-5268.

31. Ren J, Zhang S, Kovacs A, Wang Y, Muslin AJ. Role of p38alpha MAPK in cardiac apoptosis and remodeling after myocardial infarction. J Mol Cell Cardiol. 2005;38:617-623.

32. Zhu J, Gu H, Lv X, Yuan C, Ni P, Liu F. LINC-PINT activates the mitogen-activated protein kinase pathway to promote acute myocardial infarction by regulating miR-208a-3p. Circ J. 2018;82:27832792.

33. Mitra A, Ray A, Datta R, Sengupta S, Sarkar S. Cardioprotective role of P38 MAPK during myocardial infarction via parallel activation of $\alpha$-crystallin B and Nrf2. J Cell Physiol. 2014;229:1272-1282. 